\title{
OH OBSERVATIONS NEAR THE REFLECTION NEBULAE NGC 2068 AND NGC 2071
}

L. E. B. JOHANSSON and B. HÖGLUND

Onsala Space Observatory, Onsala, Sweden

A. WINNBERG

Max-Planck-Institut für Radioastronomie, Bonn, F.R.G.

NGUYEN-Q-RIEU

Observatoire de Meudon, Meudon, France

and

W. M. GOSS

Kapteyn Astronomical Laboratory, Groningen, The Netherlands

\begin{abstract}
Narrow $\mathrm{OH}$ emission lines at $1667 \mathrm{MHz}$, apparently from a Class I source, have been observed near the reflection nebula NGC 2071. The region contains many T Tauri stars. OH emission corresponding to the dust cloud north and east of NGC 2024 is also seen. At $1720 \mathrm{MHz}$ the dust cloud component appears in absorption; presumably the isotropic $2.7 \mathrm{~K}$ cosmic background is being absorbed.
\end{abstract}

(Summarized by the Editors.)

\section{Dust Cloud Emission}

We surveyed $\mathrm{OH}$ emission in the direction of the dust cloud near NGC 2024 (W12 or Orion B) between limits of $-2.0<\delta<+1.5,5^{\mathrm{h}} 40^{\mathrm{m}}<\alpha<5^{\mathrm{h}} 50^{\mathrm{m}}$, using the 25 -m radio telescope of the Onsala Space Observatory.

We found dust cloud emission at $\sim 9 \mathrm{~km} \mathrm{~s}^{-1}$ over the whole survey area north and east of NGC 2024. The antenna temperatures were $0.10 \mathrm{~K}$ at $1665 \mathrm{MHz}$ and $0.21 \mathrm{~K}$ at $1667 \mathrm{MHz}$. Because of uncertainties in gain and noise fluctuations we cannot rule out the LTE radio, but the satellite lines show clear evidence for nonthermal emission. The $1612 \mathrm{MHz}$ line is stronger than the $1665 \mathrm{MHz}$ line, and the $1720 \mathrm{MHz}$ line appears in absorption. Presumably the $2.7 \mathrm{~K}$ isotropic background is being absorbed; the situation is similar to $\mathrm{H}_{2} \mathrm{CO}$ absorption in dark clouds. A concentration of $\mathrm{T}$ Tauri stars in the vicinity may provide near-IR flux to pump the $\mathrm{OH}$ molecules to the nearest vibrationally excited state, enhancing both the $1612 \mathrm{MHz}$ emission and the $1720 \mathrm{MHz}$ absorption.

\section{The Class I Source}

We found unusual $\mathrm{OH}$ emission, apparently from a Class I source, near the reflection nebula NGC 2071. A narrow emission line appears at $1667 \mathrm{MHz}$ only. It has two components, at 13.45 and $13.75 \mathrm{~km} \mathrm{~s}^{-1}$. The half-power widths are $0.3 \mathrm{~km} \mathrm{~s}^{-1}$, implying a kinetic temperature of $\sim 33 \mathrm{~K}$ if due to thermal broadening. We saw no 
time variation between August and December 1972. We measured its position at Nançay with a beamwidth of $3.5 \times 18^{\prime}: \alpha=5^{\mathrm{h}} 44^{\mathrm{m}} 30^{\mathrm{s}} \pm 1^{\mathrm{s}}, \delta=+0^{\circ} 20^{\prime} .5 \pm 1^{\prime}(1950)$. Its galactic designation is $\mathrm{OH} 205.1-14.1$.

The Class I source is quite close to a group of T Tauri stars but does not seem to coincide with any one of them. We used the 100-m telescope at Effelsberg to search for a compact $\mathrm{H}$ il region near the source and found none to limits of $0.08 \mathrm{Jy}$ at 2.695 $\mathrm{GHz}$ and $0.02 \mathrm{Jy}$ at $10.7 \mathrm{GHz}$. We suggest the source may represent an early phase in the development of a $\mathrm{T}$ Tauri star.

We give a fuller account in Astrophysical Journal 189 (1974), 455.

L. E. B. Johansson

B. Höglund

Onsala Space Observatory,

S-430 34 Onsala, Sweden

\author{
A. Winnberg \\ Max-Planck-Institut für Radioastronomie, \\ Auf dem Hügel 69, \\ 53 Bonn 1, F.R.G. \\ Nguyen-Q-Rieu \\ Observatoire de Meudon, \\ 92 Meudon, France
}

W. M. Goss

Kapteyn Astronomical Laboratory,

Postbus 800, Groningen 8002, The Netherlands

\title{
DISCUSSION
}

Turner: The relative line brightnesses shown on the slide are consistent with near-IR pumping $(2.8 \mu \mathrm{m})$ but not far-IR pumping. These lines were said to arise over an extended area, probably a dust cloud. Are there enough T Tauri stars over this area to maintain near-IR pumping over the entire area? Are the relative line brightnesses constant over this entire area? It is important to decide these questions for dark dust clouds in general, to explain the many similar $\mathrm{OH}$ anomalies found in these clouds by Turner and Heiles (1971) and by Turner (1973).

Höglund: Herbig and Kuhi listed $45 \mathrm{~T}$ Tauri stars in the area, so I think there will be sufficient $2.8 \mu \mathrm{m}$ flux available. We observed all four ground state lines only at the position of the Class I source. 